Libérer et écologiser les sciences sociales (Foyer Jean, ISCC)

Version longue de l'article publié dans Hemes n60, Edgar Morin aux risques d’une pensée libre.

Introduction :

Edgar Morin est en passe d'être élevé au rang de " vieux sage » dans un paysage intellectuel français à la recherche de figures crédibles. Pourtant, cette reconnaissance étonnamment tardive semble encore très partielle au sein même du milieu officiel où Morin a produit l'essentiel de son œuvre : celui des sciences sociales et plus particulièrement de la sociologie française. En effet, parmi mes professeurs, collègues sociologues ou chercheurs en sciences sociales français, j'ai presque systématiquement rencontré un certain étonnement lorsque j'ai fait référence aux écrits d'Edgar Morin comme l'une des inspirations fondamentales de mon travail. Cet étonnement peut être de différents ordres : la plupart d'entre eux admet connaître mal cet œuvre débordant peut-être trop les frontières du social, certains la dédaignent de manière automatique, par réflexe corporatiste et/ou disciplinaire, d'autres enfin la connaissent et peuvent même l'admirer, mais la jugent souvent trop hermétique et difficilement mobilisable dans le cadre d'analyse sociologique. C'est peut-être essentiellement à ces derniers que s'adresse cet article qui vise à montrer qu'au-delà de certaines de leurs aspérités, les travaux de Morin peuvent être très fructueux pour n'importe quel chercheur en science social. En fait, cet article veut répondre à deux questions assez simples et pratiques : en quoi la pensée d'Edgar Morin peut-elle être utile à un (jeune) chercheur en science sociale ? En quoi cette pensée peut-elle être particulièrement féconde pour un pan spécifique des sciences sociales qui s'intéresse aux questions environnementales?

La première question fera l'objet d'une réponse personnelle, à partir de ma propre expérience de jeune sociologue dans I'utilisation pratique des travaux de Morin. La seconde question appelle une réponse plus théorique, à partir des contributions de Morin à un des débats fondamentaux de la pensée occidentale et qui anime plus que jamais les sciences sociales actuellement, celui d'un nécessaire dépassement du dualisme entre nature et culture et de ses implications drastiques pour la transformation des sciences sociales.

I. Une sociologie sans école, des sciences sociales sans disciplines

Du fait de son amplitude et de son ouverture disciplinaire et humaine, la lecture d'une œuvre comme celle de Morin est forcément partielle et personnelle. J'aimerais donc ici faire partager une lecture subjective de ce que je retiens des travaux de Morin et qui, je pense, peut être utile à d'autres chercheurs en science sociales, dans l'optique d'une libération profonde de ces dernières.

En ce qui concerne le domaine de la sociologie, il est clair que celle que pratique Morin ne répond à aucun des courants qui ont structuré cette discipline ces 50 dernières années. Morin semble complètement l'assumer tout en regrettant que ces propositions sociologiques n'aient pas rencontré un plus large écho: "Evidemment, en sociologie, j'ai toujours été à la marge des tendances dominantes. Il y a eu la tendance dite de la sociologie américaine avec des questionnaires qui portaient sur des échantillons, j'ai fait tout à fait autre chose. Puis, il y a eu une domination bourdivine, avec un déterminisme strict qui évidemment ne me convenait pas. Puis, il y a eu un moment donné un marxisme dogmatique dont j'étais à la marge aussi. Touraine, c'est un peu différent, car on trouve dans son travail l'idée de mouvement social, de sujet, il y a quelque chose de 
dynamique. (...) Qu'il y ait quelques sociologues ici où là en France qui ont suivi mes idées sur la sociologie de l'évènement ou l'auto-éco organisation, c'est vrai. Mais mes partisans sont très disséminés et très marginaux. " (Entretien personnel, 29/08/2007). Un exemple qui illustre cette marginalité est la manière tardive et relativement fortuite dont j'ai découvert Morin et l'idée de complexité, en lisant un article d'une anthropologue mexicaine ( $\mathrm{Paz}, 1996)$ pour mon mémoire de DEA (Master 2). Qu'un étudiant en sciences sociales fasse un détour du côté de l'Amérique Latine ${ }^{1}$ et n'entende parler de Morin pour la première fois qu'après 5 ans d'études supérieures en dit assez long sur la place qui lui est reconnu dans les institutions d'enseignement supérieur françaises. En ne proposant pas une méthode sociologique "clé en main " autour de quelques concepts et d'une grille de lecture applicable à n'importe quel "objet sociologique", mais plutôt un changement de paradigme épistémologique, Morin semble ne pas faire vraiment partie du jeu des "écoles " si prégnant dans le paysage académique français. Je retiens donc de Morin qu'on peut s'émanciper des écoles sociologiques et entretenir un rapport à la théorie relativement libre. A propos de son rapport à la théorie sociologique, il explique d'ailleurs : " Je suis un peu comme une abeille qui va butiner des fleurs tout à fait différentes pour faire son miel. Je trouve toujours quelque chose, même dans les sociologies auxquelles je suis rétif, s'il y a des documents intéressants, je les prends " (entretien personnel, 29/08/2007). Pour ma part et dans mon travail, je traduis concrètement cette approche pragmatique par un certain anarchisme théorique et méthodologique (Feyerabend, 1979) qui autorise à un " bricolage " scientifique, dans l'esprit de la logique de la pensée sauvage décrite par Levi-Strauss. Autour de l'épistémologie de Morin, il ne me semble pas choquant de faire cohabiter dans des mêmes analyses des auteurs français comme Bourdieu, Latour, Touraine, (pour ne citer qu'eux) réputés peu compatibles probablement plus pour des raisons institutionnelles que strictement théoriques. On peut se permettre ainsi un emprunt variable à leurs grandes métaphores du social selon les aspects que l'on veut mettre en avant. On pourra donc parler aussi bien de " champs ", de "réseaux ", de " mouvements ", de "collectifs " ou même de "système complexe " afin de souligner respectivement la relative autonomie d'un secteur social traversé de rapports de domination, mettre en avant les effets de circulation entre éléments ontologiquement divers, insister sur certaines mobilisations dans leur historicité, dépasser l'analyse des seuls humains ou enfin qualifier les dynamiques d'interactions entre éléments. Un tel parti pris peut présenter évidemment l'avantage de ses inconvénients à savoir, du côté des inconvénients, l'absence de la garantie d'une certaine solidité qu'assure le recours aux grandes architectures, testées et approuvées par les normes de qualité sociologiques françaises, puis éprouvées sur une multitudes d'objets. Du côté des avantages, on retiendra la souplesse et l'adaptabilité pragmatique du bricolage à des objets mouvants, avec surtout le plaisir d'assembler des éléments hétéroclites qui n'étaient pas forcément destinés à s'emboîter. Sur des objets hybrides et des terrains mexicains souvent accidentés comme les miens, ce syncrétisme théorique s'avère souvent plus fécond.

Ce refus des écoles au sein même de la sociologie se situe logiquement en aval du refus beaucoup plus fondamental de tout enfermement disciplinaire. A la question de savoir dans quelle mesure il assume l'ancrage disciplinaire de la sociologie, Morin répond « Cette étiquette de sociologue est tout à fait partielle. J'ai fait ma carrière au sein du CNRS au département de sociologie mais, dès le début,

\footnotetext{
${ }^{1}$ L'œuvre de Morin est probablement plus reconnue dans les milieux universitaires latino-américains des sciences sociales qu'en France, probablement du fait d'une certaine compatibilité culturelle pour des pensées syncrétiques. Pour un entretien de Morin centré sur ses rapports à l'Amérique Latine, on renvoie au dossier que la revue Espaces Latinos lui a consacré (Espaces Latinos, 2007)
} 
j'ai fait des choses qui ont intégré la sociologie tout en la débordant toujours, dans une vision que je dirai poly-disciplinaire ou transdisciplinaire, en fonction de l'objet de ma recherche. Pour mon premier livre important, L'Homme et la Mort, j'ai dû faire appel à l'ethnographie, à la sociologie des religions, à l'histoire, à la psychologie, à la psychanalyse et, bien entendu, à la biologie. (...). Je suis plutôt victime d'un monde où l'on a besoin d'être étiqueté socio-professionnellement car, malheureusement, je fais partie des personnes qui n'entrent pas dans ces classifications. " Pour ma part, je traduirais cette approche par un "sociologue, oui, mais pas trop " où, sans pour autant renier un ancrage disciplinaire fondamental, on doit évidemment puiser dans les théories et méthodes des autres sciences sociales et établir des collaborations avec les chercheurs d'autres disciplines. La transdisciplinarité que propose Morin a largement été commentée et tend à faire de plus en plus consensus, au moins dans les déclarations, elle n'en reste pas moins loin d'être évidente à mettre en œuvre et extrêmement exigeante. Elle consiste à décentrer ou plutôt déplacer constamment son regard, à rendre son point de vue le plus mobile possible pour faire varier les éclairages autour d'un même objet. II ne s'agit pas d'une simple succession de perspectives puisque ces variations de regards contribuent à redessiner de manière dynamique les contours d'un objet dont la géométrie devient variable. C'est ce que j'ai essayé de faire avec les OGM en essayant de comprendre les différentes perspectives biologiques, juridiques, économiques, sociologiques et anthropologiques qui pouvaient les éclairer, ceci pour mieux saisir les regards des différents acteurs qui prenaient part à la controverse sur cette évolution technologique (Foyer 2008; 2010). Des acteurs opposés ou aux visions différentes peuvent avoir le sentiment de débattre d'une même problématique alors qu'ils parlent depuis des sphères de représentations si différentes qu'ils ne peuvent pas toujours vraiment se comprendre. Ainsi, pour un biotechnologue, le maïs transgénique est avant tout une plante dans laquelle on a transféré un transgène issu d'un génome d'un autre organisme ; pour un semencier, un exploitant agricole ou un économiste, c'est un produit susceptible de dégager des profits ; pour un écologue, c'est un organisme d'un nouveau type interagissant avec son environnement; pour un agronome, une nouvelle variété pour la production agricole; pour un juriste, une nouvelle technologie qu'il faut réguler et, pour un paysan indigène, une atteinte morale contre un être sacré, etc.. Evidemment, ce type d'approche transdisciplinaire, mais aussi nécessairement multi-acteurs, multi-niveau et multi-située pose de sérieux problèmes pratiques dans sa mise en œuvre, que ce soit au niveau de la collecte ou de celui du traitement des données. II oblige à sélectionner drastiquement des sources très différentes, à multiplier les terrains et types d'informateurs (scientifiques, politiques, membres de la société civile,...), à comprendre différents langages, parfois techniques, ou encore à articuler les niveaux d'analyses dans des synthèses transversales. Ce n'est donc qu'au prix d'un certain surcout de travail que l'on peut plonger au cœur de la complexité mais là encore, l'épistémologie de la complexité proposée par Morin est une aide des plus précieuses dans cette entreprise. La théorisation de la complexité, essentiellement présentée dans les différents tomes de la Méthode, représente très certainement la colonne vertébrale de l'œuvre de Morin. Ce sont ces réflexions qui permettent d'éviter les écueils d'une pensée simple, structurée autour des piliers d'ordre, de séparabilité, de réduction et de la raison inductive/déductive (Morin et Le Moigne, 1999 ; p. 112-118). Si la pensée de Morin érode les césures disciplinaires, elle demande en retour une certaine discipline pour se départir des habitus de notre raison simple et adopter des automatismes de pensée complexe. A mon sens, l'un des plus productifs de ces réflexes consiste à s'efforcer de penser comment deux ou plusieurs éléments sont à la fois concurrents, contradictoires et complémentaires. Ce principe dialogique, en admettant qu'une interrelation puisse prendre simultanément des états différents, à la manière des ondes- 
corpuscules de la physique quantique, permet de prendre en compte une gamme de possibles plus ample et d'éviter par-là les analyses trop univoques. Appliquée au dualisme nature/culture dont on va parler plus avant, la dialogie impliquerait que nature et culture sont en lutte perpétuelle (les humains détruisent la nature en même temps que celle-ci peut les détruire comme le tremblement de terre Japonais et ses suites nous le prouvent), qu'elles sont en tension compétitive constante (l'emprise de l'homme sur la nature à travers les biotechnologies est concomitante de la menace globale d'un réchauffement climatique), mais également qu'elles co-évoluent selon des dynamiques symbiotiques (les hommes produisent de la Nature en même temps que la Nature les produit). On reviendra sur ce type de réflexions dans la deuxième partie de ce texte mais on doit conclure cette première partie sur ce qu'on pourrait appeler la force libératrice des propositions de Morin.

Une sociologie sans école, des sciences sociales sans disciplines et une méthode qui serait en fait une a-méthode (Morin, 1977, p 15-16), Morin ne nous proposerait-il pas une lecture des sciences sociales critiques, libertaires et anarchistes, à la manière d'un Ivan llich à propos des institutions médicales ou éducatives? Comme toujours dans la pensée de Morin, les choses sont plus complexes: sa portée critique est radicale et profonde mais elle ne se limite pas à cette dimension et propose de véritables alternatives théoriques et outils pratiques. On a également vu que son rejet des disciplines requiert une certaine discipline de pensée et que le refus des écoles est loin d'être une facilité théorique. Si la pensée de Morin libère, c'est des dogmes, des habitudes et des facilités de penser en ouvrant les différentes pièces et en abattant les principales cloisons de la maison des sciences sociales. Non content des résultats encore partiels de ces travaux de rénovation interne, Morin propose d'aérer également cette maison des sciences sociales en créant des ouvertures et des percées dans ses murs, en direction des autres sciences, notamment celles du vivant.

\section{Ecologiser les sciences sociales}

Que ce soit à partir de l'anthropologie de la nature (Descola, 2005), de la sociologie des sciences (Latour, 1991;1999) de l'éthique de l'environnement (Larrère et Larrère, 1997) ou d'une sociologie plus générale s'interrogeant sur le sens social de la crise écologique (Beck, 2000), la problématique classique du clivage moderne entre nature et société tend à s'imposer come une des interrogations les plus fondamentales, mais aussi théoriquement les plus fécondes, des sciences sociales ces dernières années. Non seulement cette question est politiquement fondamentale du fait de l'urgence de penser une crise écologique dont les effets transforment en profondeur le tissu social, mais elle est non moins essentielle du point de vue épistémologique, puisqu'elle interroge les limites de l'objet même des sciences sociales : la société. Penser la nature depuis les sciences sociales amène donc inévitablement à repenser radicalement ces dernières. Elles ne peuvent plus considérer l'environnement comme un simple décor inerte et passif puisque il devient de plus en plus évident que la nature (rétro) agit constamment avec le social. Comment dès lors faire rentrer cette nature en science sociale et reconsidérer un des impensés majeurs de ces sciences sociales modernes ? Les pistes de réflexion pour répondre à ce problème sont nombreuses mais la contribution de Morin à ce débat est particulièrement ambitieuse et stimulante.

Elle repose sur une ouverture aux différentes sciences du vivant, alors même que la tradition des sciences sociales françaises se montre très rétive face au soupçon du moindre déterminisme 
biologique ${ }^{2}$. Pour Morin, la meilleure manière de s'opposer à ce type de doctrines et à leurs possibles dérives n'est certainement pas de nier une nature dont les effets de feed-back sont de plus en plus évidents, ni de se réfugier dans le constructivisme social abusif que les STS (Science and Technology Studies) ont pu parfois adopter en considérant la nature comme un simple construit social. C'est au contraire en essayant de se plonger dans les sciences du vivant, en écoutant ce qu'elles ont à nous dire, en tentant d'en comprendre les concepts fondamentaux et les logiques épistémiques pour mieux se les réapproprier que Morin cherche à retisser les liens coupés. Son séjour au Salk Institute de San Diego à la fin des années 60 aux côtés des plus grands chercheurs des sciences du vivant, au moment où la biologie moléculaire constitue ses fondements théoriques et la révolution culturelle hippie explose ${ }^{3}$, est probablement déterminant dans la « biologisation » de la pensée de Morin. Les propriétés auto -organisatrices du vivant sont notamment au cœur de la pensée complexe qui, à l'opposé d'un structuralisme qui tendait à figer des objets dans des structures stables, est une pensée organique, au sens où elle est instable et animée, à la manière d'une entité vivante. Des molécules jusqu'à l'univers, en passant par les sociétés animales et humaines ou la biosphère, la capacité d'auto-production et d'auto-génération (auto-poïesis) est omniprésente dans les idées de Morin. C'est notamment dans le tome 2 de la Méthode (la Vie de la Vie) que cette exploration de I'auto-organisation est la plus profonde. Au niveau philosophique et social, ces caractéristiques du vivant peuvent se traduire par les qualités de subjectivité et d'autonomie en réaction à l'objectivisme et aux déterminismes stricts. Morin prend bien soin d'éviter tout holisme radical en reconnaissant toujours cette autonomie aux différentes entités qu'il analyse. L'ADN dans la cellule, la cellule dans l'organisme, l'organisme dans l'individu, l'individu dans la société, la société humaine dans les sociétés animales et, plus largement, dans la biosphère, toutes ces entités fonctionnent, jusqu'à un certain point, de manière autonome. D'un point de vue des sciences sociales et de leur objet, la société et les hommes, cette vision a des implications bien évidemment drastiques. Tout d'abord, la société humaine, si elle a bien des caractéristiques propres (notamment du fait de la spécificité cérébral des homos sapiens sapiens), n'en fonctionne pas moins selon les mêmes principes fondamentaux d'auto-organisation que les autres entités vivantes. Le social et l'humain se voient donc reconnectés dans le continuum vivant sans pour autant s'y réduire et par là même, les entités biologiques se retrouvent dotées d'une autonomie, voire d'une subjectivité propre. Dès lors, personnifier la biosphère dans l'hypothèse Gaïa (Lovelock, 1999) ou reconnaitre un certain type de subjectivité et de sociabilité aux non humains (animaux, plantes,...) comme le font depuis toujours les sociétés traditionnelles (Descola, 2005) n'apparait plus impossible. L'organisation sociale, la culture ou la subjectivité ne sont donc plus forcément le monopole et le privilège des êtres humains. L'humain n'est pas réduit au biologique, mais bien réintroduit dans la sphère du vivant. Si cela rentre en collision avec notre anthropocentrisme moderne, Morin semble ouvrir la voie à des sciences sociales animistes, reconnaissant la spécificité humaine tout autant que son inscription dans un continuum biologique.

\footnotetext{
${ }^{2}$ Le darwinisme social de Spencer, sa possible récupération politique dans des thèses eugénistes et racistes, et le moindre soupçon de retour du déterminisme biologique sous les formes par exemple de la sociobiologie (Wilson, 1975), semblent paralyser la très républicaine tradition sociologique française quant à la possibilité de parler d'un autre objet que la société.

${ }^{3}$ Sur cette période, on ne peut que conseiller la lecture du Journal de Californie où Morin mélange très librement ses réflexions épistémologiques avec ses observations sociologiques sur l'extrême occident que représente la Californie (Morin, 1970).
} 
L'indépendance relative de l'auto-organisation a pour pendant dialogique l'interdépendance écosystémique. Les autonomies n'ont en effet de sens que par rapport aux interdépendances d'ensemble. La science écologique et son concept central d'écosystème trouvent donc une résonnance particulière dans l'épistémologie (Morin, 1980, pp. 19-96) et la pensée du social (Morin, 1994 pp. 117-152 ; Morin, 2007) de Morin. Morin retient de l'écologie scientifique le centrage sur les différents types d'interdépendances (parasitisme, symbiose, prédation, hiérarchie,...), la relation entre les vivants (biocénose) et le milieu physique (biotope), la mobilisation de savoirs divers, et, évidemment l'approche systémique. Néanmoins, cette approche systémique ne se veut pas restreinte à une niche particulière, mais au contraire profondément ouverte. Morin perçoit ainsi les limites d'une écologie odumienne ${ }^{4}$ classique qui tend à percevoir l'homme comme un perturbateur et donc, à l'exclure, ainsi qu'à mythifier l'état de climax comme un état stable et optimum (Larrère et Larrère, 1997). Les socio-écosystèmes de Morin sont ouverts comme dans l'écologie des paysages et ils intègrent également les éléments humains. Loin de mythifier la stabilité, Morin prend en compte les évènements et les désordres comme dans l'écologie des perturbations. Des sciences sociales écologisées ne renvoient donc pas à une sociologie de l'environnement, comme il existe par exemple une sociologie du travail ou encore des médias, au sens où elle ne désigne pas seulement un objet particulier d'étude. Elle ne consiste, en effet, pas seulement à étudier les acteurs sociaux ancrés dans le champ de l'environnement (ONG, experts, populations locales...), même si cette dimension est importante, mais bien aussi à étudier écologiquement la société, c'est-à-dire selon une approche systémique et intégrale qui met l'accent sur les dynamiques de lien, en premier lieu entre les sphères sociale et naturelle. L'objet " environnement » a donc changé le regard sociologique en l'écologisant. Ce changement de perspective cadre clairement avec la volonté de contribuer à des évolutions épistémologiques contre une certaine hyperspécialisation scientifique, et en faveur de ce que Beck appelle une spécialisation sur le lien (Beck, 2001). C'est ce que semble aussi signifier Descola et Pálsson quand ils invitent à dépasser certains dualismes fondamentaux, en premier lieu celui entre nature et culture pour que " s'ouvre un paysage intellectuel entièrement différent où les états et les substances sont remplacés par les processus et les relations » (Descola et Pálsson, 1994). Dès lors, caractériser et décrire finement les différents types de relations plutôt que les objets sociaux devient la tâche centrale du chercheur en sciences sociales.

Ce retournement épistémologique s'accompagne chez Morin d'un bouleversement éthique et politique puisque il en appelle avec force à l'émergence d'une conscience écologique. Dès 1972, il écrit « C'est toute l'idéologie occidentale de Descartes qui faisait l'homme sujet dans un monde d'objets, qu'il faut renverser. C'est l'idéologie de l'homme unité insulaire, monade close dans l'univers, contre quoi le romantisme n'a pu réagir que poétiquement, contre quoi le scientisme n'a pu réagir que mécaniquement en faisait de l'homme une chose, lui aussi. Le capitalisme et le marxisme ont continué à exalter " la victoire de l'homme sur la nature » comme si c'était l'exploit le plus épique que d'écrabouiller la nature. Cette idéologie des Cortés et Pizaro de l'écosystème conduit en fait au suicide, la nature vaincue, c'est l'autodestruction de l'homme " (Morin, 2007, p15.). Cette prise de conscience écologique se traduit au niveau politique par la mise en évidence de la gravité de la crise écologique, mais également par les critiques des idéologies du développement infini, du consumérisme, de la toute-puissance de la techno-science, de l'aveuglement économique ou encore

\footnotetext{
${ }^{4}$ Si la notion d'écosystème a été définie par Tansley dans les années 30, la synthèse de l'outillage conceptuel (écosystème, biocénose, biotope, réseau trophique,...) des sciences écologiques a été réalisée par les frères Odum dans un ouvrage de 1953.
} 
du règne de la quantité sur la qualité. En cela, l'œuvre de Morin entre également en résonnance avec celle des pionniers de l'écologie politique comme Ellul, Illich, Dumont ou Gorz. Comme pour ces penseurs, l'écologie politique de Morin s'inscrit néanmoins dans une pensée politique plus large, en l'occurrence, en faveur d'une politique de civilisation qu'il appelle de ses vœux. Ecologiser les sciences sociales et, plus largement, la pensée représente donc à la fois pour Morin un programme épistémologique et politique, qui consiste à prendre le contre-pied radical de Descartes. Quand celuici voulait diviser le monde pour mieux que l'homme tout puissant puisse y régner en maître, Morin propose au contraire de remembrer notre vision de l'univers naturel et social pour mieux cohabiter.

Conclusion :

Libérer des carcans académiques et disciplinaires, ouvrir à d'autres horizons théoriques, relier ce qui semble disjoint, réanimer et faire bouger des sciences sociales figées et recluses dans leur territoire : tels sont les enseignements principaux que je retiens de mes lectures de Morin. La vertu première de ces lectures est certainement de stimuler l'intuition à l'origine de la créativité scientifique. En guise de conclusion, je me permets donc de proposer une des intuitions inspirées par Morin, qui a été au cœur de mon travail ces dernières années (Foyer, 2008 ; 2010) et qui mériterait, pour être consolidée, de devenir un programme de travail à part entière pour les prochaines années. On peut la résumer ainsi : de la même manière que la méthode de Descartes correspondrait à l'ère moderne de la séparabilité (Modernité simple), on peut penser que celle de Morin correspond aux évolutions en cours de cette modernité, marquée par le phénomène de globalisation (Modernité globale). II s'agit avec cette intuition d'essayer d'articuler les théories sur la complexité, la modernité " avancée » et la globalisation. Elle suppose de redéfinir la modernité essentiellement par sa méthode mais aussi d'élargir la définition de la globalisation, non plus entendue comme synonyme de mondialisation ${ }^{5}$, mais comme un méta-processus d'intégration, un élan complexificateur où se multiplient les interactions de toutes sortes, notamment entre les sphères politiques, économiques, scientifiques, techniques et environnementales. Cette globalisation-complexification serait en quelque sorte une vision dynamique et historicisée de l'idée de complexité.

Bibliographie :

Beck, Ulrich. La société du risque. Sur la voie d’une autre modernité. Paris : Aubier, 2001. 525p.

Descola, Philippe. Par-delà nature et culture. Paris : Gallimard, 2005. 623p.

Descola, Philipe et Pálsson, Gisli. (Ed.) Nature and society: Anthropoligical perspectives. London : Routledge, 1996. 310p.

Espaces Latinos. Edgar Morin et l'Amérique Latine, n²43, 2007. pp. 17-26

Foyer, Jean. Diversité naturelle et culturelle face aux défis des biotechnologies : enjeux et controverses au Mexique. Thèse de doctorat de sociologie (Dir. Yvon Le Bot), Institut des Hautes Etudes de l'Amérique Latine - Paris III Sorbonne Nouvelle, 2008. 658p. Disponible sur : http://tel.archives-ouvertes.fr/tel-00545542/fr/

Foyer, Jean. Il était une fois la bio-révolution: nature et savoirs dans la modernité globale. Paris : Presses Universitaires de France, 2010. 220p.

\footnotetext{
${ }^{5}$ Dans cette optique, la mondialisation n'est en fait que le versant territorial de la globalisation.
} 
Larrère, Catherine et Larrère, Raphaël. Du bon usage de la nature : Pour une philosophie de I'environnement. Paris : Aubier, 1997. 360p.

Latour, Bruno. Nous n'avons jamais été modernes - essai d'anthropologie symétrique, Paris : La Découverte/Poche, 1997 (Paris : La Découverte 1991). 200p.

Latour, Bruno. Politiques de la nature. Comment faire entrer les sciences en démocratie. Paris : La Découverte/Poche, 2004 (Paris : La Découverte, 1999). 375p.

Lovelock, James. La terre est un être vivant : I'hypothèse Gaïa. Paris : Champs Flammarion, 1999. $183 p$.

Morin, Edgar. Journal de Californie. Paris : Seuil, 1970. 268p.

Morin, Edgar. La méthode, 1. La Nature de la Nature. Paris : Seuil, 1977. 400p.

Morin, Edgar. La méthode, 2. La Vie de la Vie. Paris : Seuil, 1980. 470p.

Morin, Edgar. Sociologie. Paris : Fayard, 1994. 470p.

Morin, Edgar. L’an I de l'ère écologique. Paris : Talandier, 2007. 127p.

Morin, Edgar et Le Moigne, Jean Louis. L'intelligence de la complexité. Paris : L'Harmattan, 1999. 330p.

Paz, María Fernanda. La dimensión cultural de la problemática ambiental. In Iztapalapa (México), vol.16, n40, 1996. pp. 163-184 